\title{
Fullerene Thermochemical Stability: Accurate Heats of Formation for Small Fullerenes, the Importance of Structural Deformation on Reactivity, and the \\ Special Stability of $\mathrm{C}_{60}$
}

Bun Chan*

ABSTRACT: We have used quantum chemistry computations, in conjunction with isodesmictype reactions, to obtain accurate heats of formation (HoFs) for the small fullerenes $\mathrm{C}_{20}$, $\left(2358.2 \pm 8.0 \mathrm{~kJ} \mathrm{~mol}^{-1}\right), \mathrm{C}_{24}(2566.2 \pm 7.6)$, and the lowest-energy isomers of $\mathrm{C}_{32}(2461.1 \pm$ 15.4), $C_{42}(2629.0 \pm 20.5)$ and $C_{54}(2686.2 \pm 25.3)$. As part of this endeavor, we have also obtained accurate HoFs for several medium-sized molecules, namely $216.6 \pm 1.4$ for fulvene, $375.5 \pm 1.5$ for pentalene, $670.8 \pm 2.9$ for acepentalene, and $262.7 \pm 2.5$ for acenaphthylene. We combine the energies of the small fullerenes and previously obtained energies for larger fullerenes (from $\mathrm{C}_{60}$ to $\mathrm{C}_{6000}$ ) into a full picture of fullerene thermochemical stability. In general, the per-carbon energies can be reasonably approximated by the " $R+D$ " model that we have previously developed (J. Chem. Theory Comput. 2019, 15, 1255-1264), which takes

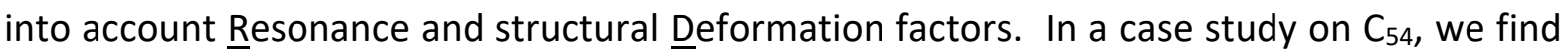
that most of the high-deformation-energy atoms correspond to the sites of $\mathrm{C}-\mathrm{Cl}$ bond in the experimentally captured $\mathrm{C}_{54} \mathrm{Cl}_{8}$. In another case study, we find that $\mathrm{C}_{60}$ has the lowest value for the maximum local-deformation-energy when compared with similar-sized fullerenes, which is consistent with its "special stability". These results are indicative of structural deformation playing an important role in the reactivity of fullerenes. 


\section{INTRODUCTION}

Fullerenes are a poster child of modern chemistry. ${ }^{1}$ As a "new" allotrope of carbon discovered after diamond and graphite, it has expanded the horizon of what is possible with just one element. Unlike diamond and graphite, fullerenes are individual molecules; there is potentially infinite number of discrete fullerenes, each has its own unique properties. The scale of possibilities associated with fullerenes has led to abundant researches in their fundamental chemistry and physics, ${ }^{2,3}$ as well as in their applications to various fields of technology. ${ }^{4,5}$ To this day, fullerenes continue to be a focus of active scientific research. ${ }^{6}$

Our own interest in fullerenes involves research into their thermochemistry. In a series of studies, ${ }^{7-9}$ we have used large-scale computational quantum chemistry to obtain accurate heats of formation (HoFs) for fullerenes ranging from $C_{60}$ to $C_{320}$. The large number of these accurate data has enabled us to subsequently devise and validate a theoretically motivated model (termed " $R+D$ ") for rapid estimation of fullerene HoFs. ${ }^{10}$ We have shown that this nearly-cost-free model provides good accuracy for the estimated HoFs for $\sim 3500$ fullerenes up to $\mathrm{C}_{6000}$. Notably, our model reveals that the thermochemical stability of medium-sized fullerenes (from $\mathrm{C}_{60}$ to $\sim \mathrm{C}_{100}$ ) depends on a delicate balance of resonance and deformation factors. This finding suggests the possibility of tuning the isomeric structure of fullerenes for different applications. In another study, we have also briefly extended our investigation to cover both thermochemical and kinetic stabilities of fullerenes. ${ }^{9}$ In passing, we note some closely related contributions to this area by others. ${ }^{11-15}$

While our studies have provided a practical means for estimating HoFs for $\mathrm{C}_{60}$ and larger fullerenes, we have yet to undertake a detail investigation into a series of fullerenes that are smaller than $\mathrm{C}_{60}$, nor are we aware of any study to this effect. At this point, let us recall that the discovery of $\mathrm{C}_{60}$ is aided by it having substantially higher stability than both larger and smaller fullerenes. ${ }^{16}$ If we look at this fact from another angle, it implies that both larger and smaller fullerenes exhibit higher reactivity than $C_{60}$, and these higher reactivities may be advantageous in some chemical applications.

In the present study, we use a range of computational chemistry methods, from high-order coupled cluster to the R+D model, to examine in detail the HoFs of small fullerenes from $C_{20}$ onwards. Our aim is to first provide reliable HoFs for these systems, and to use these values together with chemical models to uncover the underlying components for the thermochemical stability of these fullerenes. By comparing their thermochemistry with those 
of medium-sized and large fullerenes, we hope to gain new insights that may in due course contribute to new fullerene chemistry.

\section{COMPUTATIONAL METHODS}

Standard quantum chemistry calculations were carried out with Gaussian 16, ${ }^{17}$ Molpro 2019, ${ }^{18}$ MRCC, $^{19}$ and Q-Chem 5. ${ }^{20}$ Lists of fullerenes and their initial structures were generated with the FULLERENE program, ${ }^{21}$ which was also employed to obtain topological resonance energies. ${ }^{22,23}$ We used a local implementation of the local-curvature model of ref 24 to obtain energies of deformation of the fullerenes, as we did previously. ${ }^{10}$ Unless otherwise noted, geometries, zero-point vibrational energies (ZPVE), and thermal corrections for $298 \mathrm{~K}$ enthalpies $\left(\Delta H_{298-0}\right)$, were obtained with the B3-LYP ${ }^{25}$ density functional theory (DFT) method in conjunction with the cc-pVTZ basis set, ${ }^{26}$ as specified in high-level WnX-type protocols. ${ }^{27-31}$ Vibrational frequencies were examined to affirm the nature of the optimized geometries as minima on the potential energy surfaces. Literature scale factors were applied to the frequencies in the calculation of ZPVE (0.9886) and $\Delta H_{298-0}(0.9926) .{ }^{32,33}$ Improved single-point energies were computed using a variety of methods that include, notably, the high-level W1X-127 and W3X-L $\mathrm{L}^{29}$ composite protocols, and the $\mathrm{B} 97 \mathrm{M}-\mathrm{V}^{34}$ and $\mathrm{MN} 15^{35} \mathrm{DFT}$ methods (with the maug-cc-pVTZ ${ }^{36}$ basis set). Other methods that are discussed in the text include the W1X-2, ${ }^{27}$ WG, ${ }^{31} \mathrm{G} 4(\mathrm{MP} 2)-6 \mathrm{X},{ }^{37,38}$ and CBS-QB33 ${ }^{39}$ composite procedures, as well as the DuT-D3 ${ }^{40}$ double-hybrid DFT method. Unless otherwise noted, energies in the text represent $298 \mathrm{~K}$ enthalpies in $\mathrm{kJ} \mathrm{mol}^{-1}$.

\section{RESULTS AND DISCUSSION}

Preparation for Obtaining Heat of Formation for $\mathbf{C}_{\mathbf{2 0}}$. The caged $\mathrm{C}_{20}$ molecule is the smallest fullerene, i.e., the smallest carbon cage that includes twelve pentagon rings. While each carbon atom in $\mathrm{C}_{20}$ is formally $s p^{2}$ hybridized, the small-caged geometry causes significant deformation from the ideal planar structure for $s p^{2}$ carbon. One may then expect considerable partial breaking of the $\pi$-bonds in $C_{20}$. This could in turn lead to substantial "multi-reference" character and thus pose considerable challenges to even high-level quantum chemistry methods such as the "gold standard" $\operatorname{CCSD}(T)^{41}$ at the complete-basis-set (CBS) limit. Indeed, this has been suggested in a recent investigation. ${ }^{42}$ On the other hand, another study argues that the $C_{20}$ cage does not possess significant multi-reference character so long as its structure is not in the icosahedral $\left(I_{h}\right)$ symmetry. ${ }^{43}$ In that study, the energies for several non- $I_{h} C_{20}$ structures are found to be similar, and all of them are lower in energy 
than $I_{h} C_{20}$. The observation that the non- $I_{h} C_{20}$ molecules have similar energies is consistent with a study ${ }^{44}$ in which a CCSD(T)/CBS-type method is used. In the present study, we also find that our geometry optimization leads to a non- $/ \mathrm{h}$ structure, specifically, $C_{2 \mathrm{~h}}$.

While some of the aforementioned studies hint that good energy for $C_{20}$ can be obtained using a generally good method such as CCSD(T)/CBS, no higher-level method has been applied to $C_{20}$ to definitively put an end to this matter. With the prohibitive cost of post-CCSD(T) computations, which generally limits their application to systems with just a hand-full of atoms, we do not envisage that we can tackle this issue head-on using existing resources. Nonetheless, it would be reassuring to gain further confidence in methods that are applicable to $\mathrm{C}_{20}$. As we have discussed earlier, we perceive that a potential source of complication for quantum chemistry methods is the structural deformation to the formally $s p^{2}$ carbon atoms in $\mathrm{C}_{20}$ cage. We have thus investigated this issue using small model systems.

Coping with Structural Deformation. We first examine ethene as the smallest prototypical system with a formal $\mathrm{C}=\mathrm{C}$ double bond. Specifically, we have systematically probed the energetic consequence for distorting its geometry from planarity. The structures that we used are obtained from a series of constrained optimizations (Figure 1). As an example, for ethene, we define two dummy atoms $(X)$ and constrain the $X-C-H$ angles to be $90^{\circ}$. Thus, $X-$ $\mathrm{C}$ is perpendicular to the corresponding plane of $\mathrm{H}-\mathrm{C}-\mathrm{H}$. We then vary the $\mathrm{X}-\mathrm{C}-\mathrm{C}$ angle from $90^{\circ}$, which corresponds to the equilibrium structure, to $160^{\circ}$, in $10^{\circ}$ intervals. This leads to increasingly distorted "bowl-shaped" structures that resemble those in $\mathrm{C}_{20}$ cage. All other geometrical parameters are fully optimized.
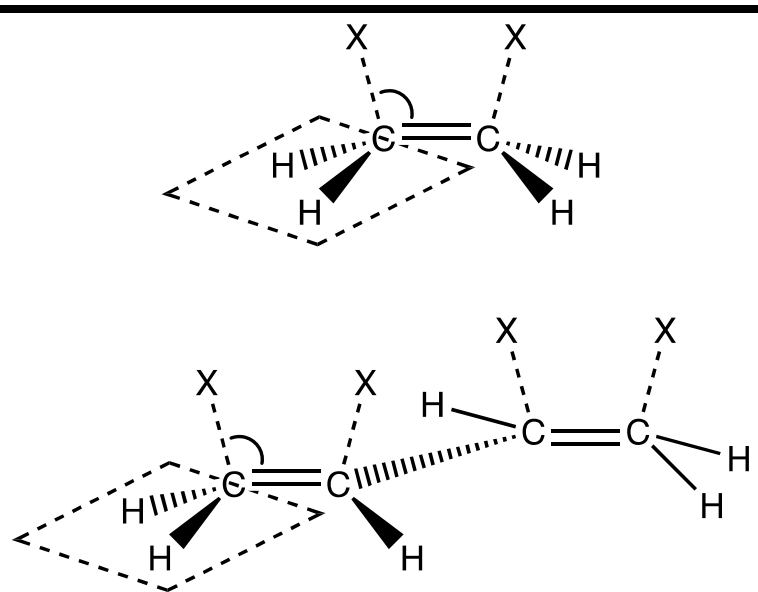

Figure 1. Systems used to assess quantum chemistry methods for coping with structural deformations similar to those in caged $\mathrm{C}_{20}$, with $\mathrm{X}$ representing a dummy atom. As an example, 
for ethene, the $\mathrm{X}-\mathrm{C}-\mathrm{H}$ angles are held constant at $90^{\circ}$ such that each $\mathrm{X}-\mathrm{C}$ is perpendicular to the relevant $\mathrm{H}-\mathrm{C}-\mathrm{H}$ plane. The $\mathrm{X}-\mathrm{C}-\mathrm{C}$ angles are widened from $90^{\circ}$ in $10^{\circ}$ intervals.

The highest-level protocol that we use to obtain the energies is, in the spirit of the W4 method ${ }^{45}$ that is among the most accurate quantum chemistry procedures, a composite method (CM) that includes post-CCSD(T) contributions up to $\operatorname{CCSDTQ}(5)$. It is defined by:

$$
\begin{aligned}
& E_{\mathrm{CM}}=E_{\mathrm{HF} / \mathrm{A}^{\prime} \mathrm{V}[5,6] \mathrm{Z}}+\Delta E_{\mathrm{CCSD} / \mathrm{A}^{\prime} \mathrm{V}[5,6] \mathrm{Z}}+\Delta E_{\mathrm{CCSD}(\mathrm{T}) / \mathrm{A}^{\prime} \mathrm{V}[\mathrm{Q}, 5] \mathrm{Z}} \\
& +\Delta E_{\mathrm{CCSDT} / \mathrm{N}[\mathrm{T}, \mathrm{O}] \mathrm{Z}^{*}}+\Delta E_{\mathrm{CCSDT}(\mathrm{Q}) / \mathrm{N}[\mathrm{D}, \mathrm{T}] \mathrm{Z}^{*}} \\
& +\Delta E_{\mathrm{CCSDTQ} / \mathrm{V}[\mathrm{D}, \mathrm{T}] \mathrm{Z}^{*}}+\Delta E_{\mathrm{CCSDTQ}(5) / \mathrm{NDZ}^{*}} \\
& +\Delta \operatorname{CR} \operatorname{ccsD}(\mathrm{T}) / \mathrm{CV}[0,5] \mathrm{Z}
\end{aligned}
$$

in which $\Delta E_{C C S D}$ indicates the energy of CCSD minus that for its immediately lower-level method, which is Hartree-Fork (HF) in the case of CCSD, and so on. Unless otherwise noted, we apply the frozen-core approximation to all correlation computations. The notation $[D, T] Z$ signifies CBS extrapolation using the DZ (double- $\zeta$ ) and TZ (triple- $\zeta$ ) basis sets, and so on. In all cases, we use the extrapolation formula $E_{L}=E_{\mathrm{CBS}}+A L^{-\alpha}, 46$ in which $L$ corresponds to the basis set such that $L=2$ for DZ, etc. The value of $\alpha$ is 5 for extrapolating HF energies, and it is 3 for all other components.

The generic "*" designation indicates reduced-sized basis sets in line with our computational resources. For the applicable components, they are defined as:

$$
\begin{aligned}
& \Delta E_{\mathrm{CCSDT} / \mathrm{V}[\mathrm{T}, \mathrm{Q}] \mathrm{Z}^{*}:} \quad \mathrm{VTZ} * \text { includes } s p \text { functions for } \mathrm{H} \text { and } s p d \text { functions for } \mathrm{C} \\
& \text { VQZ* includes spd functions for } \mathrm{H} \text { and spdf functions for } \mathrm{C}
\end{aligned}
$$

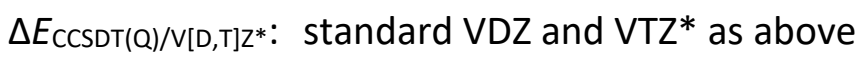

$$
\begin{aligned}
& \Delta E_{\mathrm{CCSDTQ} / \mathrm{V}[\mathrm{D}, \mathrm{T}] Z^{*}:} \quad \text { VDZ* includes } s \text { functions for } \mathrm{H} \text { and } s p \text { functions for } \mathrm{C} \\
& \text { VTZ* includes VTZ } s \text { functions and VDZ } p \text { functions for } H \text {, } \\
& \text { and VTZ sp functions and VDZ } d \text { functions for } C \\
& \Delta E_{\mathrm{CCSDTQ}(5) / \mathrm{VDZ}^{*}:} \quad \text { VDZ } \mathrm{s} \text { functions for } \mathrm{H} \text { and standard VDZ for } \mathrm{C}
\end{aligned}
$$

Finally, the $\triangle \mathrm{CR}$ term represents a single component for the combined core-correlation plus scalar-relativistic effects. It is defined as the energy from an all-electron Douglas-KrollHess $^{47,48}$ computation minus the energy from the corresponding frozen-core non-relativistic calculation.

The benchmark energies for ethene relative to the equilibrium structure (i.e., deformation $=0^{\circ}$ ) are shown in Figure 2 (top), together with those obtained with several progressively 
lower-level methods. These methods include W3X-L, which is a cost-effective high-level protocol that contains post-CCSD(T) effects up to CCSDT(Q), the W1X-1, WG and G4(MP2)-6X methods, which are progressively more economical approximations to CCSD(T)/CBS, and DuTD3, a double-hybrid density functional theory (DH-DFT) method designed to be used in conjunction with a moderate triple- $\zeta$ basis set. They represent a set of more efficient methods that may serve as a secondary benchmark.
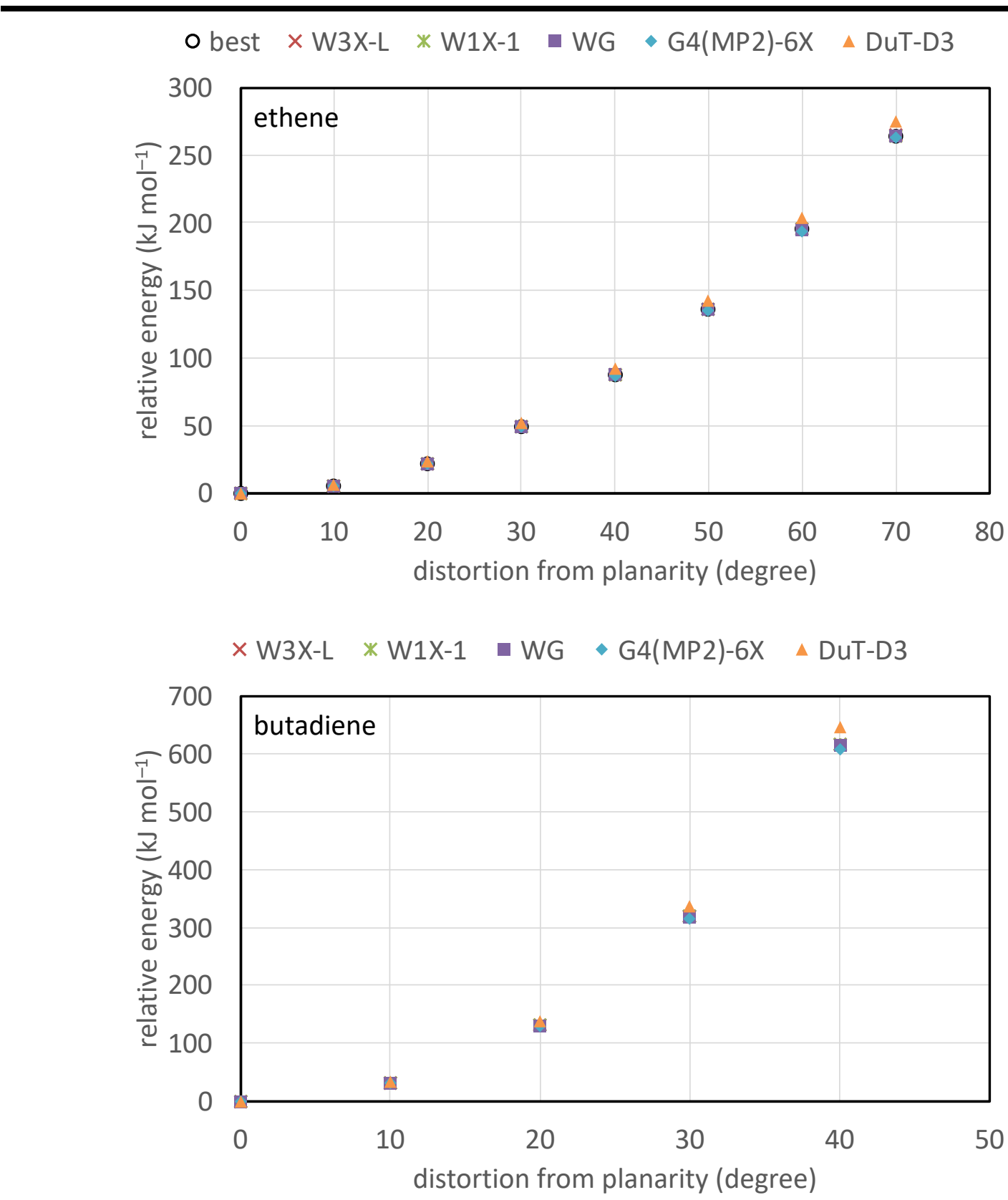

Figure 2. Ethene and butadiene energies relative to those at equilibrium structures for those with increasingly deformed geometries according to Figure 1 . The designation "best" refers to our highest-level protocol defined in the "Coping with Structural Deformation" section. 
We can see that all methods yield relative energies that are fairly comparable to one another for the entire range of distorted structures, which include a maximum deformation angle of $70^{\circ}$ and an associated relative energy of $+264.2 \mathrm{~kJ} \mathrm{~mol}^{-1}$ at our benchmark level. The deviations from benchmark energies are somewhat larger for DuT-D3 than those for other methods. In all cases, the point of largest deformation shows the largest deviations, with values of +0.3 [W3X-L], -0.2 [W1X-1], -0.3 [WG], -1.2 [G4(MP2)-6X] and +10.9 [DuT-D3] kJ mol $^{-1}$, respectively. Overall, it is (pleasantly) surprising that these lower-level methods all handle such deformations reasonably well, even at the furthest end of the scale.

For butadiene, the most deformed structure corresponds to a deformation angle of just $40^{\circ}$ (versus $70^{\circ}$ for ethene); larger deformations would lead to steric crowding of the hydrogen atoms. Nonetheless, at the W3X-L level, which represents our highest-level method that is computationally feasible, the most deformed structure has a relative energy of +615.1 $\mathrm{kJ} \mathrm{mol}^{-1}$, which more than doubles that for ethene $\left(+264.2 \mathrm{~kJ} \mathrm{~mol}^{-1}\right)$. We again see that all methods cope with such structural deformations reasonably well. The deviations from the W3X-L benchmark energies at the deformation angle of $40^{\circ}$ are +0.3 [W1X-1], -2.1 [WG], 8.6 [G4(MP2)-6X], and +31.4 [DuT-D3] kJ mol-1. While many of these deviations are more substantial than those for ethene, W1X-1 still holds up well with sub-kJ mol ${ }^{-1}$ deviations throughout the entire range of distorted structures. We thus deem both W3X-L and W1X-1 to be adequate in handling structural deformations like the ones in $C_{20}$ fullerene.

Heat of Formation for $\mathbf{C}_{\mathbf{2 0}}$. We now proceed to the computation of the HoF for $\mathrm{C}_{20}$ using isodesmic-type reactions. In order to maximize the accuracy in the computed reaction energies, one would ideally use large molecular motifs of $C_{20}$ in the isodesmic-type reactions. This would provide better cancelation of systematic errors, as well as minimization of the number of species involved in the reactions, and hence minimization of associated uncertainties. In practice, however, large molecular species are often associated with large uncertainties in their HoF values. For the computation of unknown HoF values, this would erode the improved accuracies associated with the calculated reaction energies.

For $\mathrm{C}_{20}$, a key structural motif is represented by acepentalene $\left(\mathrm{C}_{10} \mathrm{H}_{6}\right)$, which is bowlshaped and consists of three fused five-membered rings that resemble faces of $C_{20}$. However, we are not aware of an accurately known HoF value for acepentalene. Smaller fragments of $\mathrm{C}_{20}$ can be described with pentalene $\left(\mathrm{C}_{8} \mathrm{H}_{6}\right.$, two fused five-membered ring) and fulvene $\left(\mathrm{C}_{6} \mathrm{H}_{6}\right.$, a five-membered ring plus an exocyclic $=\mathrm{CH}_{2}$ group). In a recent study, ${ }^{42}$ the HoFs for 
pentalene and fulvene have been obtained using a combination of high-level quantum chemistry composite protocols, bond-separation reactions, and statistically derived empirical corrections. The values are $374.9 \pm 5.4$ (pentalene) and $215.5 \pm 4.2$ (fulvene) $\mathrm{kJ} \mathrm{mol}^{-1}$.

While the uncertainties associated with the pentalene and fulvene HoFs are not excessive, they are still notably larger than those for smaller fragments for which highly accurate HoFs from Active Thermochemical Tables (ATcT) ${ }^{49}$ are available. For example, applying bondseparation reactions to $\mathrm{C}_{20}$ would require ethene $\left(\mathrm{H}_{2} \mathrm{C}=\mathrm{CH}_{2}\right)$, ethane $\left(\mathrm{H}_{3} \mathrm{C}-\mathrm{CH}_{3}\right)$ and methane $\left(\mathrm{CH}_{4}\right)$, and their ATcT HoF values are $52.36 \pm 0.12,-83.97 \pm 0.13$, and $-74.526 \pm 0.055 \mathrm{~kJ} \mathrm{~mol}^{-}$ ${ }_{1}^{1}$, respectively. Notably, ATcT HoF is also available for the larger butadiene molecule $\left(\mathrm{C}_{4} \mathrm{H}_{6}\right.$, $110.83 \pm 0.37 \mathrm{~kJ} \mathrm{~mol}^{-1}$ ), which can also be used in isodesmic-type reactions for $\mathrm{C}_{20}$. We note that ATcT HoF is available for benzene, which shares the same molecular formula with fulvene. However, the six-membered benzene ring does not represents a structural motif of $C_{20}$, and we deem its use for $\mathrm{C}_{20}$ isodesmic-type reactions not satisfactory. To obtain the $\mathrm{C}_{20} \mathrm{HoF}$, we thus come down to the two bond-separation-type reactions:

$$
\begin{aligned}
& \mathrm{C}_{20}+40 \mathrm{CH}_{4} \rightarrow 10 \mathrm{C}_{2} \mathrm{H}_{4}+20 \mathrm{C}_{2} \mathrm{H}_{6} \\
& \mathrm{C}_{20}+30 \mathrm{CH}_{4} \rightarrow 5 \mathrm{C}_{4} \mathrm{H}_{6}+15 \mathrm{C}_{2} \mathrm{H}_{6}
\end{aligned}
$$

Let us briefly digress and consider the likely uncertainties associated with the HoFs obtained from these two reactions. In each case, the total uncertainty in the $\mathrm{C}_{20} \mathrm{HoF}$ has two components, namely the uncertainty in the calculated reaction energy and the uncertainties associated with the HoFs of the constituent species. The latter can be obtained straightforwardly using the quoted ATcT uncertainties together with standard errorpropagation principle. ${ }^{50}$ Regarding the former, in our previous study, ${ }^{8}$ we have examined the works of Wheeler et al. ${ }^{51}$ on a large set of isodesmic-type reactions. We have inferred that a conservative estimate of uncertainty would be $0.2 \mathrm{~kJ} \mathrm{~mol}^{-1}$ per species when the energy for a well-balanced isodesmic-type reaction is obtained using a high-level method such as $\operatorname{CCSD}(\mathrm{T}) / \mathrm{CBS}$. The two components can then be combined using error propagation to yield the total uncertainty.

If we now apply the W1X-1 method to the two bond-separation reactions together with the method for estimating errors as detailed above, we obtain $\mathrm{C}_{20} \mathrm{HoF}$ values of $2364.5 \pm 14.2$ $\mathrm{kJ} \mathrm{mol}^{-1}$ for the "ethene reaction" and $2355.9 \pm 10.3 \mathrm{~kJ} \mathrm{~mol}^{-1}$ for the "butadiene reaction". In comparison, if we use the atomization approach to calculate $\mathrm{C}_{20} \mathrm{HoF}$, we arrive at a value of $2366.2 \mathrm{~kJ} \mathrm{~mol}^{-1}$. In general, the atomization approach is testing for quantum chemistry 
methods, such that low-level methods often yield significantly different HoFs when compared with those obtained using isodesmic-type reactions. ${ }^{51}$ The reasonable agreement between our "atomization HoF" and the two "isodesmic HoFs" provides additional support to the adequacy of W1X-1 used in our calculations.

To further substantiate our computed $\mathrm{C}_{20} \mathrm{HoF}$, we turn to recent literature. In ref 42 , a value of $2359.8 \pm 15.9 \mathrm{~kJ} \mathrm{~mol}^{-1}$ has been obtained, and both of our values, with the associated uncertainties, overlap with this reported HoF. We also note that, their quoted uncertainty is obtained on the basis of analyzing an independent set of bond-separation reactions. The agreement between our uncertainty of $14.2 \mathrm{~kJ} \mathrm{~mol}^{-1}$ and the reported value of $15.9 \mathrm{~kJ} \mathrm{~mol}^{-1}$ provides a cross validation in the methods for error estimation. Finally, the availability of our two values enables us to bracket the $\mathrm{C}_{20} \mathrm{HoF}$. By this means, we arrive at our recommended value of $2358.2 \pm 8.0 \mathrm{~kJ} \mathrm{~mol}^{-1}$.

Choosing a Method for the Computation of Larger Fullerenes. For fullerenes that are larger than $\mathrm{C}_{20}$, the application of the high-level W1X-1 protocol would rapidly become unfeasible. We have thus assessed a series of less-costly methods in order to achieve adequate accuracies in the computation of HoFs for larger fullerenes. Our assessment employs a series of relevant isodesmic-type reactions of small unsaturated hydrocarbons. Specifically, they include the following bond-separation reactions:

$$
\begin{aligned}
& \mathrm{C}_{4} \mathrm{H}_{6} \text { (butadiene) }+2 \mathrm{CH}_{4} \rightarrow 2 \mathrm{C}_{2} \mathrm{H}_{4}+1 \mathrm{C}_{2} \mathrm{H}_{6} \\
& \mathrm{C}_{6} \mathrm{H}_{6} \text { (fulvene) }+6 \mathrm{CH}_{4} \rightarrow 3 \mathrm{C}_{2} \mathrm{H}_{4}+3 \mathrm{C}_{2} \mathrm{H}_{6} \\
& \mathrm{C}_{8} \mathrm{H}_{6} \text { (pentalene) }+10 \mathrm{CH}_{4} \rightarrow 4 \mathrm{C}_{2} \mathrm{H}_{4}+5 \mathrm{C}_{2} \mathrm{H}_{6} \\
& \mathrm{C}_{10} \mathrm{H}_{6} \text { (acepentalene) }+14 \mathrm{CH}_{4} \rightarrow 5 \mathrm{C}_{2} \mathrm{H}_{4}+7 \mathrm{C}_{2} \mathrm{H}_{6} \\
& \mathrm{C}_{20}+40 \mathrm{CH}_{4} \rightarrow 10 \mathrm{C}_{2} \mathrm{H}_{4}+20 \mathrm{C}_{2} \mathrm{H}_{6}
\end{aligned}
$$

as well as bond-separation-type reactions using progressively larger molecular fragments, such as $\mathrm{C}_{8} \mathrm{H}_{6}+6 \mathrm{CH}_{4} \rightarrow 2 \mathrm{C}_{4} \mathrm{H}_{6}+3 \mathrm{C}_{2} \mathrm{H}_{6}$ and $\mathrm{C}_{8} \mathrm{H}_{6}+4 \mathrm{CH}_{4} \rightarrow 1 \mathrm{C}_{6} \mathrm{H}_{6}+1 \mathrm{C}_{2} \mathrm{H}_{4}+2 \mathrm{C}_{2} \mathrm{H}_{6}$. In addition, we are able to compute the $C_{24}$ fullerene with W1X-1, and have included in our test set the two bond-separation-type reactions:

$$
\begin{aligned}
& \mathrm{C}_{24}+8 \mathrm{CH}_{4} \rightarrow 1 \mathrm{C}_{20}+2 \mathrm{C}_{2} \mathrm{H}_{4}+4 \mathrm{C}_{2} \mathrm{H}_{6} \\
& \mathrm{C}_{24}+6 \mathrm{CH}_{4} \rightarrow 1 \mathrm{C}_{20}+1 \mathrm{C}_{4} \mathrm{H}_{6}+3 \mathrm{C}_{2} \mathrm{H}_{6}
\end{aligned}
$$

In total, there are 17 bond-separation-type reactions in our test set, which we will refer to as the BSR17 set (signifying Bond-Separation Reaction). We have used BSR17 to evaluate the accuracy of a collection of 18 lower-level methods. Selected statistical results are shown in 
Table 1, while the full set of statistical indicators are provided in the Supporting Information. We can see that, the W1X-2 method, which is closely related to W1X-1, performs quite well. Its mean absolute deviation (MAD) for this set of reactions is just $2.9 \mathrm{~kJ} \mathrm{~mol}^{-1}$. If we divide each absolute deviation by the number of species involved in the reaction, and consider all reactions, we can arrive at an average MAD per species (MAD/n) of $0.1 \mathrm{~kJ} \mathrm{~mol}^{-1}$. The largest deviation of $+8.5 \mathrm{~kJ} \mathrm{~mol}^{-1}$ occurs for the reaction $\mathrm{C}_{20}+40 \mathrm{CH}_{4} \rightarrow 10 \mathrm{C}_{2} \mathrm{H}_{4}+20 \mathrm{C}_{2} \mathrm{H}_{6}$. It involves a total of 71 species and thus corresponds to an absolute deviation per species of $0.1 \mathrm{~kJ} \mathrm{~mol}^{-}$ 1 , which coincides with the MAD/n value.

Table 1. Mean Absolute Deviation (MAD, kJ mol-1) from Benchmark W1X-1 Values, Mean Deviation (MD), Standard Deviation of the Deviations (SD), and Largest Deviation (LD), for the BSR17 Set of 17 Bond-Separation-Type Reactions of Unsaturated Hydrocarbons

\begin{tabular}{lcccc} 
method & MAD & MD & SD & LD \\
B97M-V & 13.6 & -0.5 & 16.9 & -33.3 \\
MN15a & 13.8 & -7.6 & 19.2 & -41.5 \\
DuT-D3 & 13.5 & +4.4 & 18.2 & +38.0 \\
CBS-QB3 & 23.2 & +21.6 & 28.1 & +75.8 \\
G4(MP2)-6X & 12.1 & +12.1 & 12.8 & +36.4 \\
WG & 10.0 & +10.0 & 10.8 & +32.8 \\
W1X-2 & 2.9 & +2.9 & 2.1 & +8.5 \\
a The maug-cc-pVTZ basis set is used. & & & \\
\hline
\end{tabular}

In comparison with W1X-2, lower-level methods are considerably less accurate for this set of systems. Notably, the WG, G4(MP2)-6X and CBS-QB3 methods all represent reasonably high-level composite protocols that are generally quite accurate. For instance, for a set of over 800 diverse thermochemical quantities, the MADs for W1X-2, WG and G4(MP2)-6X are 2.2, 2.3 and $3.6 \mathrm{~kJ} \mathrm{~mol}^{-1}$, respectively. ${ }^{31}$ A key difference between $\mathrm{W} 1 \mathrm{X}-1$ and $\mathrm{W} 1 \mathrm{X}-2$ on the one hand, and the lower-level composite protocols on the other hand, is that the two W1X methods employ larger basis sets for the $\operatorname{CCSD}(T)$ computations. We thus infer that the reactions in the BSR17 set have fairly strong requirements for the basis sets in the high-level wavefunction correlation calculations.

Having seen MADs of more than $10 \mathrm{~kJ} \mathrm{~mol}^{-1}$ for the lower-level composite protocols, the performance for the B97M-V, MN15, and DuT-D3 methods then comes as a pleasant surprise. 
The MADs for the three methods are 13.6 (B97M-V), 13.8 (MN15), and 13.5 (DuT-D3) kJ mol1 , which are comparable with that for the G4(MP2)-6X method $\left(12.1 \mathrm{~kJ} \mathrm{~mol}^{-1}\right)$. With that being said, there are also cases with large deviations ( $L D \sim 40 \mathrm{~kJ} \mathrm{~mol}^{-1}$ in magnitude). Further analysis shows that B97M-V and MN15 perform comparably with one another, while DuT-D3 is somewhat less reliable for the relevant $\mathrm{C}_{20}$ and $\mathrm{C}_{24}$ species (Supporting Information, Table S2). We find that averaging the B97M-V and MN15 reaction energies produces more robust results, especially for the fullerene reactions (Table S2). In addition, the B97M-V and MN15 methods also fall under the class of computationally most efficient methods that we have examined, and they can be straightforwardly applied to fairly large systems. We thus deem it most appropriate to use these two methods as a cost-effective means for calculating energies of the larger fullerenes in the present study.

Managing Uncertainties for Larger Fullerenes. Between $\mathrm{C}_{20}$ and $\mathrm{C}_{60}$, there are 18 distinctsized sets of fullerenes, with $\mathrm{C}_{24}$ being the smallest one. Rather than exhaustively sample all of them, we have investigated $C_{24}, C_{32}, C_{42}$ and $C_{54}$ as a set that coarsely spans the range between $C_{20}$ and $C_{60}$. As noted earlier, we have obtained W1X-1 energy for $C_{24}$, and this is used to obtain $\mathrm{C}_{24} \mathrm{HoF}$ in conjunction with the two previously mentioned bond-separationtype reactions in the BSR17 set, i.e., $\mathrm{C}_{24}+8 \mathrm{CH}_{4} \rightarrow 1 \mathrm{C}_{20}+2 \mathrm{C}_{2} \mathrm{H}_{4}+4 \mathrm{C}_{2} \mathrm{H}_{6}$ and $\mathrm{C}_{24}+6 \mathrm{CH}_{4} \rightarrow$ $1 \mathrm{C}_{20}+1 \mathrm{C}_{4} \mathrm{H}_{6}+3 \mathrm{C}_{2} \mathrm{H}_{6}$. The HoF values are $2567.2 \pm 8.6 \mathrm{~kJ} \mathrm{~mol}^{-1}$ for the former and $2565.5 \pm$ $8.3 \mathrm{~kJ} \mathrm{~mol}^{-1}$ for the latter reactions, which lead to our recommended valued of $2566.2 \pm 7.6$ $\mathrm{kJ} \mathrm{mol}^{-1}$ by bracketing the ranges spanned by the two values and their uncertainties.

For those larger fullerenes, we find that the use of both W1X-1 and W1X-2 methods to be computationally unattainable. We thus apply the average of B97M-V and MN15 to the computation of their HoFs. Because the use of the lower-level DFT methods will inevitably contribute to larger uncertainty, we have adjusted the approach that we use in the isodesmictype reaction scheme. We recall that, for $\mathrm{C}_{20}$ and $\mathrm{C}_{24}$, we use bond-separation-type reactions to compute their HoFs. In those cases, even though there are a large number of species involved in the reactions, the use of the high-level W1X-1 method puts a lid on the total uncertainties $\left(8.0 \mathrm{~kJ} \mathrm{~mol}^{-1}\right.$ for $\mathrm{C}_{20}$ and $7.6 \mathrm{~kJ} \mathrm{~mol}^{-1}$ for $\left.\mathrm{C}_{24}\right)$. For the average of B97M-V and $\mathrm{MN15}$, the uncertainty relative to $\mathrm{W} 1 \mathrm{X}-1$ reference is $0.7 \mathrm{~kJ} \mathrm{~mol}^{-1}$ per species for the fullerene isodesmic-type reactions used in the present study (Table S2). With this additional uncertainty associated with the use of the DFT energies, the total uncertainty would rapidly balloon with increasing number of species involved in an isodesmic-type reaction. 
To reduce the nominal uncertainty in a computed DFT reaction energy, a straightforward means is to decrease the number of species in the equation. This would necessitate the use of larger species in the reaction. As mentioned earlier, for species that are of relevance as structural motifs of small fullerenes, highly accurate ATcT HoF values are available only for ethene and butadiene. Despite this, for somewhat larger species such as fulvene, pentalene, and acepentalene in the BSR17 set, we have calculated W1X-1 HoFs and the associated uncertainties using bond-separate-type reactions with ethene and butadiene. We have also obtained W1X-1 HoF values for acenaphthylene $\left(\mathrm{C}_{12} \mathrm{H}_{8}\right)$, which is relevant to the calculation of $\mathrm{C}_{54} \mathrm{HoF}$. In each case, our final recommended value is obtained by bracketing the HoFs obtained with the "ethene reaction" and "butadiene reaction", as we did with $C_{20}$ and $C_{24}$. The thus obtained HoFs are $216.6 \pm 1.4$ (fulvene), $375.5 \pm 1.5$ (pentalene), $670.8 \pm 2.9$ (acepentalene), and $262.7 \pm 2.5$ (acenaphthylene) $\mathrm{kJ} \mathrm{mol}^{-1}$. In comparison, the reported values in ref 42 are $215.5 \pm 4.2$ (fulvene), $374.9 \pm 5.4$ (pentalene), and $262.3 \pm 8.8$ (acenaphthylene) $\mathrm{kJ} \mathrm{mol}^{-1}$.

Let us now examine the uncertainties associated with the use of these larger molecules for computing HoFs of larger fullerenes, and compared them with those for reactions using smaller molecules. For $C_{32}$, an isodesmic-type reaction that employs $C_{24}$ and pentalene is:

$$
\mathrm{C}_{32}+6 \mathrm{CH}_{4} \rightarrow 1 \mathrm{C}_{24}+1 \mathrm{C}_{8} \mathrm{H}_{6}+3 \mathrm{C}_{2} \mathrm{H}_{6}
$$

The combined uncertainty related to the uncertainties in the HoFs for methane $\left(0.055 \mathrm{~kJ} \mathrm{~mol}^{-}\right.$ $\left.{ }^{1}\right)$, ethane (0.13), pentalene (1.5), and $C_{24}(7.6)$ is $7.8 \mathrm{~kJ} \mathrm{~mol}^{-1}$. If we instead replace pentalene with ethene, a balanced isodesmic-type reaction would be:

$$
\mathrm{C}_{32}+16 \mathrm{CH}_{4} \rightarrow 1 \mathrm{C}_{24}+4 \mathrm{C}_{2} \mathrm{H}_{4}+8 \mathrm{C}_{2} \mathrm{H}_{6}
$$

The uncertainties related to the HoFs of the components amount to $7.6 \mathrm{~kJ} \mathrm{~mol}^{-1}$, which is comparable to that for the pentalene reaction despite that pentalene has an uncertainty of $1.5 \mathrm{~kJ} \mathrm{~mol}^{-1}$, which is about an order of magnitude larger than that for ethene $\left(0.12 \mathrm{~kJ} \mathrm{~mol}^{-1}\right)$. The similarity between the uncertainty of $7.8 \mathrm{~kJ} \mathrm{~mol}^{-1}$ for the pentalene reaction and $7.6 \mathrm{~kJ}$ $\mathrm{mol}^{-1}$ for the ethene reaction mainly comes down to that both of them are dominated by the uncertainty of $7.6 \mathrm{~kJ} \mathrm{~mol}^{-1}$ for $\mathrm{C}_{24}$.

We now turn our attention to the uncertainty associated with the average of B97M-V and MN15 reaction energies. For the pentalene reaction, which involves a total of 12 species, the presumed uncertainty for the reaction energy is $10.2 \mathrm{~kJ} \mathrm{~mol}^{-1}$. Thus, by propagating this with the uncertainty of $7.8 \mathrm{~kJ} \mathrm{~mol}^{-1}$ for the component HoFs, we arrive at a total uncertainty of 
$12.8 \mathrm{~kJ} \mathrm{~mol}^{-1}$ for the $\mathrm{C}_{32} \mathrm{HoF}$. For the ethene reaction, the total number of species involved is 30 , which more than doubles that for the pentalene reaction. As a result, the nominal uncertainty for the ethene reaction is $25.5 \mathrm{~kJ} \mathrm{~mol}^{-1}$, and this dominates the total uncertainty of $26.6 \mathrm{~kJ} \mathrm{~mol}^{-1}$. The results thus show that it is more favourable to use larger structural motifs in the calculation of HoFs for the larger fullerenes.

Heats of Formation for Larger Fullerenes. In addition to constructing appropriate isodemstic-type reactions to reduce the uncertainties, for the larger fullerenes, another aspect to explore is the space of their structural isomers. Specifically, while there is only one isomer for $\mathrm{C}_{20}$ and $\mathrm{C}_{24}$, there are six for $\mathrm{C}_{32}, 45$ for $\mathrm{C}_{42}$, and the number of isomers then grows rapidly to 580 for $C_{54}$. For $C_{32}$ and $C_{42}$ with manageable number of isomers, we have determined the lowest-energy isomer in each case using the average of B97M-V/maug-ccpVTZ and MN15/maug-cc-pVTZ energies. Notably, for both fullerenes, the lowest-energy isomers are lower in energy than the second-lowest-energy isomers by $100 \mathrm{~kJ} \mathrm{~mol}^{-1}(109.6$ and $94.2 \mathrm{~kJ} \mathrm{~mol}^{-1}$, respectively, for $\mathrm{C}_{32}$ and $\mathrm{C}_{42}$ ).

For $\mathrm{C}_{54}$, the large number of isomers poses significant computational cost for the determination of the lowest-energy isomer, and the use of an adequate lower-level method would be desirable. In our previous study, we find B3-LYP/6-31G(d) to be quite accurate for determining relative energies of fullerenes. ${ }^{10}$ To further validate this finding, we compare the B3-LYP/6-31G(d) relative energies to those obtained with the average of B97M-V and MN15 for the isomers of $C_{42}$. We find a good correlation between the two sets of relative energies, with a coefficient of determination $\left(R^{2}\right)$ of 1.000; quantitatively, the MAD value is $9.6 \mathrm{~kJ} \mathrm{~mol}^{-}$ 1. Thus, we deem B3-LYP/6-31G(d) to be adequate for determining the lowest-energy isomer for $\mathrm{C}_{54}$. It is noteworthy that, at this level, we find the lowest-energy isomer for $\mathrm{C}_{54}$ to be significantly lower in energy than the second-lowest-energy one by $53.5 \mathrm{~kJ} \mathrm{~mol}^{-1}$.

With the considerations on uncertainty in mind (see Managing Uncertainties for Larger Fullerene section), we use the following isodesmic-type reactions to obtain the HoFs for $\mathrm{C}_{32}$, $\mathrm{C}_{42}$ and $\mathrm{C}_{54}$ :

$$
\begin{aligned}
& \mathrm{C}_{32}+6 \mathrm{CH}_{4} \rightarrow 1 \mathrm{C}_{24}+1 \mathrm{C}_{8} \mathrm{H}_{6}+3 \mathrm{C}_{2} \mathrm{H}_{6} \\
& \mathrm{C}_{32}+10 \mathrm{CH}_{4} \rightarrow 1 \mathrm{C}_{24}+1 \mathrm{C}_{6} \mathrm{H}_{6}+1 \mathrm{C}_{2} \mathrm{H}_{4}+5 \mathrm{C}_{2} \mathrm{H}_{6} \\
& \mathrm{C}_{42}+6 \mathrm{CH}_{4} \rightarrow 1 \mathrm{C}_{24}+1 \mathrm{C}_{10} \mathrm{H}_{6}+3 \mathrm{C}_{2} \mathrm{H}_{6} \\
& \mathrm{C}_{42}+10 \mathrm{CH}_{4} \rightarrow 1 \mathrm{C}_{24}+1 \mathrm{C}_{8} \mathrm{H}_{6}+1 \mathrm{C}_{2} \mathrm{H}_{4}+5 \mathrm{C}_{2} \mathrm{H}_{6} \\
& \mathrm{C}_{54}+8 \mathrm{CH}_{4} \rightarrow 1 \mathrm{C}_{24}+1 \mathrm{C}_{12} \mathrm{H}_{8}+4 \mathrm{C}_{2} \mathrm{H}_{6}
\end{aligned}
$$




$$
\mathrm{C}_{54}+10 \mathrm{CH}_{4} \rightarrow 1 \mathrm{C}_{24}+1 \mathrm{C}_{10} \mathrm{H}_{6}+1 \mathrm{C}_{2} \mathrm{H}_{4}+5 \mathrm{C}_{2} \mathrm{H}_{6}
$$

For each fullerene, the first reaction employs a single species to provide the extra unsaturated carbon atoms to the immediately smaller fullerene. For example, there is a gap of eight unsaturated carbon atoms between $\mathrm{C}_{32}$ and $\mathrm{C}_{24}$, and we use pentalene $\left(\mathrm{C}_{8} \mathrm{H}_{6}\right)$ to fill this gap. The second reaction for each fullerene replaces this "filler molecule" with an immediately smaller one plus a ethene, e.g., pentalene $\left(\mathrm{C}_{8} \mathrm{H}_{6}\right) \rightarrow$ fulvene $\left(\mathrm{C}_{6} \mathrm{H}_{6}\right)+$ ethene $\left(\mathrm{C}_{2} \mathrm{H}_{4}\right)$. This second reaction serves as a somewhat independent means for obtaining the target HoF. We note that, for each fullerene, the two values obtained from the two reactions are generally quite similar, with a difference that is usually less than $10 \mathrm{~kJ} \mathrm{~mol}^{-1}$.

Table 2. Best Estimate for Heats of Formation (HoF, $\mathrm{kJ} \mathrm{mol}^{-1}$ ) Obtained in the Present Study for the Unsaturated Hydrocarbons and Fullerenes, and Comparison with Literature Values where Data are Available

$\begin{array}{lcc}\text { species }^{\text {a }} & \text { HoF } & \text { literature }^{\mathrm{b}} \\ \mathrm{C}_{6} \mathrm{H}_{6} \text { (fulvene) } & 216.6 \pm 1.4 & 215.5 \pm 4.2,224^{\mathrm{c}} \\ \mathrm{C}_{8} \mathrm{H}_{6} \text { (pentalene) } & 375.5 \pm 1.5 & 374.9 \pm 5.4,330.1^{\mathrm{d}} \\ \mathrm{C}_{10} \mathrm{H}_{6} \text { (acepentalene) } & 670.8 \pm 2.9 & 262.3 \pm 8.8,263.2 \pm 3.7^{\mathrm{c}} \\ \mathrm{C}_{12} \mathrm{H}_{8} \text { (acenaphthylene) } & 262.7 \pm 2.5 & 2359.8 \pm 15.9 \\ \mathrm{C}_{20} & 2358.2 \pm 8.0 & \\ \mathrm{C}_{24} & 2566.2 \pm 7.6 & \\ \mathrm{C}_{32} & 2461.1 \pm 15.4 & \\ \mathrm{C}_{42} & 2629.0 \pm 20.5 & \\ \mathrm{C}_{54} & 2686.2 \pm 25.3 \\ \text { a For } \mathrm{C}_{32}, \mathrm{C}_{42} \text { and } \mathrm{C}_{54} \text {, the HoF values represent those for the lowest-energy isomers. }{ }^{\mathrm{b}} \\ \text { Literature values from ref } 42 \text { unless otherwise noted. }{ }^{\mathrm{c}} \text { NIST Chemistry Webbook. }{ }^{52}{ }^{\mathrm{d}} \text { Ref } 53 .\end{array}$

Using the combination of the two-types of isodesmic reactions and the two DFT methods (B97M-V and MN15), our proposed HoF for each of the larger fullerene is calculated as the average of four values. They are $2461.1 \pm 15.4\left(C_{32}\right), 2629.0 \pm 20.5\left(C_{42}\right)$, and $2686.2 \pm 25.3$ $\left(\mathrm{C}_{54}\right) \mathrm{kJ} \mathrm{mol}^{-1}$. We have in several recent investigations applied statistical approaches to the assessment and development of DFT methods. ${ }^{54-56}$ Using a similar concept, in the present study, we have briefly examined the use of a completely independent (statistical) approach to obtain $\mathrm{C}_{32}, \mathrm{C}_{42}$ and $\mathrm{C}_{54} \mathrm{HoFs}$ (Supporting Information). The results are similar to the above 
recommended values, which further support the perceived accuracy of our proposed values. For the convenience of the readers, we summarize in Table 2 our best estimate of all the HoF values obtained in the present study. The optimized structures of these species are provided in the Supporting Information.

Trends in Fullerene Relative Energies. With the newly obtained energies for the small fullerenes from $C_{20}$ to $C_{54}$, we can now put together a complete picture of fullerene energies by integrating them with the ones obtained previously for larger ones from $C_{60}$ to $C_{6000}$. The energies for $C_{60}$ and medium-sized fullerenes up to $C_{320}$ are taken from our previous studies, ${ }^{8,10}$ whereas those for the larger ones are from ref 57 . In accord with our previous work, we normalize the energies on a per-carbon basis to enable a direct comparison of the values for fullerenes of difference sizes. In the present study, we use $I_{h} C_{60}$ as the reference, for which we assign a relative energy of zero. The results are shown in Figure 3.

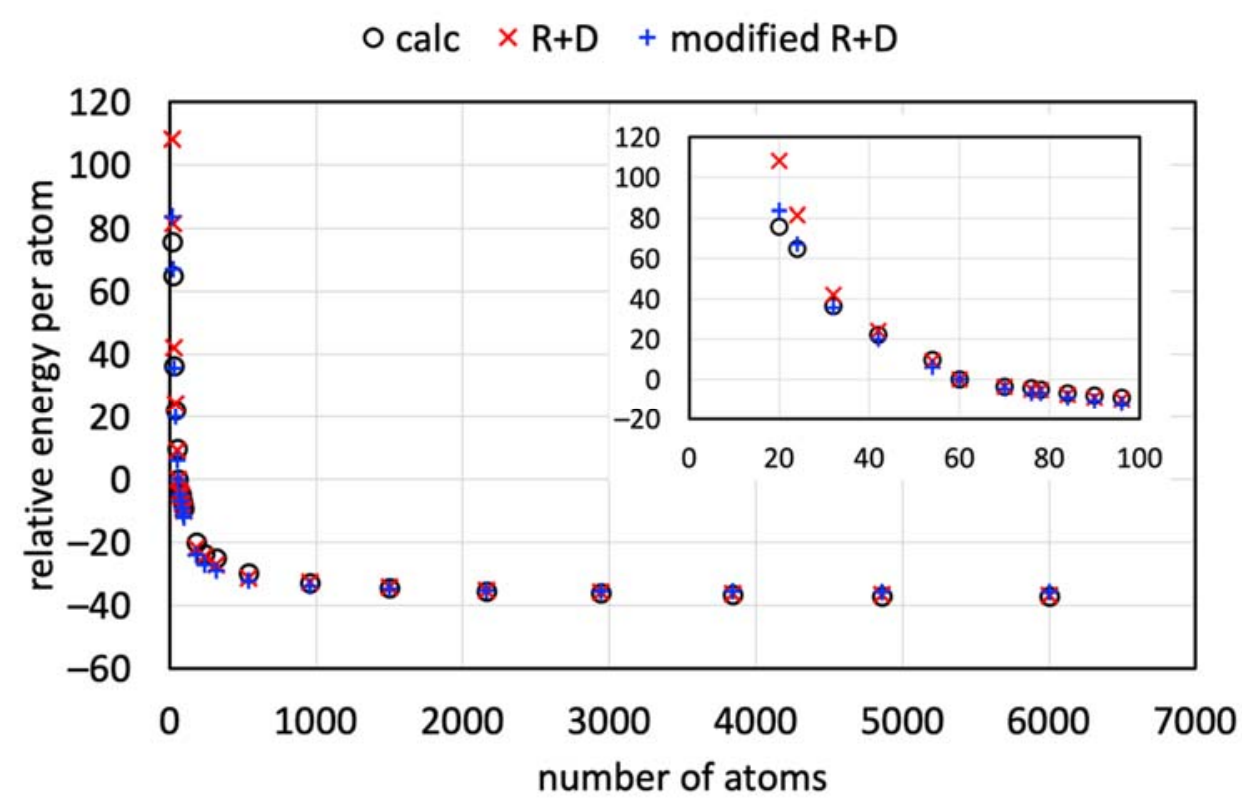

Figure 3. Relative energy per carbon $\left(\mathrm{kJ} \mathrm{mol}^{-1}\right)$ for fullerenes from $\mathrm{C}_{20}$ to $\mathrm{C}_{6000}$ obtained using quantum chemistry computations, the R+D model, and a modified R+D model. Inset shows the region between $C_{20}$ and $C_{96}$. See text and ref 10 for details of the $R+D$ model.

We can see a smooth decrease of the relative energy from $C_{20}\left(+75.9 \mathrm{~kJ} \mathrm{~mol}^{-1}\right)$ to $\mathrm{C}_{6000}(-$ $\left.37.3 \mathrm{~kJ} \mathrm{~mol}^{-1}\right)$. The trend can be approximated with the formula: $E=2338.5 \mathrm{~N}^{-1.01}-37.0 \mathrm{~kJ}$ $\mathrm{mol}^{-1}$, where $N$ is the number of carbon atoms. This represents a refined power formula than the one we previously reported ${ }^{8}$ on the basis of fitting to a subset of medium-sized fullerenes (from $\mathrm{C}_{60}$ to $\mathrm{C}_{320}$ ). We have previously shown in ref 10 that, for a large set of fullerene isomers 
from $C_{60}$ to $C_{6000}$, the relative energy per carbon can be accurately described by the R+D model, which contains a Resonance term and a Deformation term. Such an agreement is displayed in Figure 3.

For the smaller fullerenes examined in the present study, however, the agreement between the computed relative energies and the ones estimated by the $R+D$ model is not as good. In particular, the discrepancy becomes larger as the size of the fullerene decreases. This observation may indicate the existence of additional factors that stabilizes progressively smaller fullerenes. However, we reason that the relative energies for the smaller fullerenes can be accounted for with the concept behind the R+D model.

The resonance energy term in $R+D$ is obtained as the topological resonance energy within the framework of the Hückel theory. ${ }^{22}$ It describes the intrinsic differences between fullerenes of different sizes and isomeric structures that possess different connectivities of the $\pi$-bond networks in hypothetically planar fullerenes. The deformation term is derived using continuum elasticity theory and treats the total energy due to deformation from planarity as sum of local deformation energies at each carbon atom. ${ }^{24}$ The final form of this deformation term includes several approximations to facilitate straightforward calculation of the local deformations. However, if one goes back to the basis of its derivation, and make an alternative assumption that fullerenes are perfect spheres with all atoms being evenly distributed on the surface, then the deformation energy per carbon would take a simple form of $E=4 \pi D(1+\alpha) / N$. In this equation, both $D$ and $\alpha$ are constants, and as a result the percarbon deformation energy is inversely proportionally to the number of carbon atoms $N$.

We have examined the deformation energy component of the relative $R+D$ energies in Figure 3, and find that it fits to the equation: $E=3962.6 N^{-1.19}-29.0 \mathrm{~kJ} \mathrm{~mol}^{-1}$. Thus, it has a steeper inverse-power scaling with respect to $N$ than the simple inverse scaling suggested above. Let us now devise an alternative equation for the deformation energy. First, we put in place the constraint of $N^{-1}$ scaling, which then leaves the pre-factor and the constant to be determined. As the $R+D$ model provides good estimate of the trend in relative energies for $C_{60}$ to $C_{6000}$, we fit the equation to those data points, which leads to $E=1792.9 \mathrm{~N}^{-1}-29.5 \mathrm{~kJ}$ $\mathrm{mol}^{-1}$. Using this formula in conjunction with the resonance term, we obtain modified R+D energies as shown in Figure 3, with notably better agreement with the computed relative energies. Thus, a conceivable explanation for the deteriorated agreement between our 
original $R+D$ model and the calculated energies is that the simplifications made in the derivation of the deformation term become less valid as the fullerene becomes smaller.

To further refine the protocol for determining deformation energy and substantiate our hypothesis is beyond the scope of the present study. We also note that, the deformation term in the modified R+D model does not distinguish different isomers of a given-sized fullerene. We do not intent for it to be a practical alternative to the original R+D model, which performs well for a large range of medium-sized to large fullerenes, with shortcomings occurring only for the smallest fullerenes. The modified $R+D$ model is used in the present study solely as a diagnostic tool to rationalize the limitation of the original model.

Case Study 1: Reactivity of Small Fullerenes. At this point, our results suggest that the relative energy (and hence relative thermochemical stability) of the small fullerenes, like those for the larger ones, are mostly determined by the intrinsic $\pi$-bond connectivity and the degree of deformation from planarity. For fullerenes as small as $\sim C_{32}$, the magnitudes of these effects can be adequately estimated using our previously proposed R+D model. With the clearer understanding of the underlying factors that determine the energies of small fullerenes, we now briefly investigate their chemistry using the R+D model.

In our previous study, we have examined the variations in the resonance and deformation terms for a given-sized fullerene. We find that, for medium-sized fullerenes that satisfied the "isolated pentagon rule" (IPR), ${ }^{58}$ the two types of energies vary to similar degrees, as indicated by their similar standard deviation (SD) values for the per-carbon resonance energies and the per-carbon deformation energies (Table 3). However, if we include non-IPR fullerenes (denoted "gen", which signifies "general"), the deformation term shows notably larger variations (SDs) than those for resonance, for which the SD values are similar to those for the IPR fullerenes.

All fullerenes that are smaller than $\mathrm{C}_{60}$ belong to the non-IPR family, and we can see that, for $\mathrm{C}_{42}$ and $\mathrm{C}_{54}$ with statistically significant number of isomers ( 45 and 580 , respectively), the SDs are similar to those for the general sets of larger fullerenes. Thus, the degree of structural deformation is the major factor that determines the relative thermochemical stabilities of isomers for a given-sized small fullerene. Because the deformation term in the R+D model is a sum of local deformations at all atomic sites, we deem it reasonable to anticipate that the difference in these local deformations within a given fullerene isomer would lead to 
difference in local thermochemical stability, and hence a variation in reactivity. Indeed, this have been suggested in the original derivation of the formula for deformation energy. ${ }^{24}$

Table 3. Standard Deviations $\left(\mathrm{kJ} \mathrm{mol}^{-1}\right.$ ) Between the Per-Carbon Relative Energies Obtained using the R+D model and its Components for Various Fullerene Isomers ${ }^{a}$

\begin{tabular}{llcc} 
fullerene $^{\mathrm{b}}$ & $\mathrm{R}+\mathrm{D}$ & resonance & deformation \\
$\mathrm{C}_{32}$ & 4.1 & 1.7 & 2.5 \\
$\mathrm{C}_{42}$ & 2.9 & 0.7 & 2.9 \\
$\mathrm{C}_{54}$ & 3.3 & 0.6 & 2.9 \\
$\mathrm{C}_{60}$ (gen) & 3.1 & 0.6 & 2.8 \\
$\mathrm{C}_{86}$ (gen) & 2.9 & 0.4 & 2.7 \\
$\mathrm{C}_{96}$ (gen) & 3.5 & 0.6 & 3.2 \\
$\mathrm{C}_{120}$ (gen) & 2.3 & 0.3 & 2.2 \\
$\mathrm{C}_{150}$ (gen) & 1.9 & 0.2 & 1.8 \\
$\mathrm{C}_{86}$ (IPR) & 0.5 & 0.6 & 0.6 \\
$\mathrm{C}_{96}$ (IPR) & 0.6 & 0.5 & 0.8 \\
$\mathrm{C}_{120}$ (IPR) & 0.6 & 0.3 & 0.7 \\
$\mathrm{C}_{150}$ (IPR) & 0.7 & 0.2 & 0.7 \\
a Data for C & & & \\
(isolated pentagon rule) and non-IPR isomers. & & \\
\hline
\end{tabular}

Let us now use $\mathrm{C}_{54}$ as a case study on this hypothesis. For this fullerene, an isomer has been experimentally isolated by trapping with chlorine atoms, forming $\mathrm{C}_{54} \mathrm{Cl}_{8} .{ }^{59}$ The structure of the fullerene core corresponds to the lowest-energy isomer that we have found in the present study. As mentioned earlier, the second-lowest-energy $C_{54}$ isomer is higher in energy by $53.5 \mathrm{~kJ} \mathrm{~mol}^{-1}$, and this substantial difference in thermochemical stability is presumably a key factor in the experimental isolation of just one isomer. The structure of the isolated $\mathrm{C}_{54} \mathrm{Cl}_{8}$ has been determined by crystallography, and this is shown in Figure 4.

In the same figure, we also show $\mathrm{C}_{54}$ and our calculated local deformation energy expressed as a spectrum of colors, with bright red indicating atoms with the largest deformation, going through darker-colored regions towards bright green that signifies atoms with the lowest deformation energies. There are eight atoms with notably larger deformation energies than the rest. At the other end of the scale, two atoms have significantly smaller 
deformation energies than the other carbons. In this picture, we have also highlighted the eight atomic sites that correspond to the $\mathrm{C}-\mathrm{Cl}$ carbons in the experimentally isolated $\mathrm{C}_{54} \mathrm{Cl}_{8}$, and they are shown as balls, as opposed to tubes for the rest of the structure.

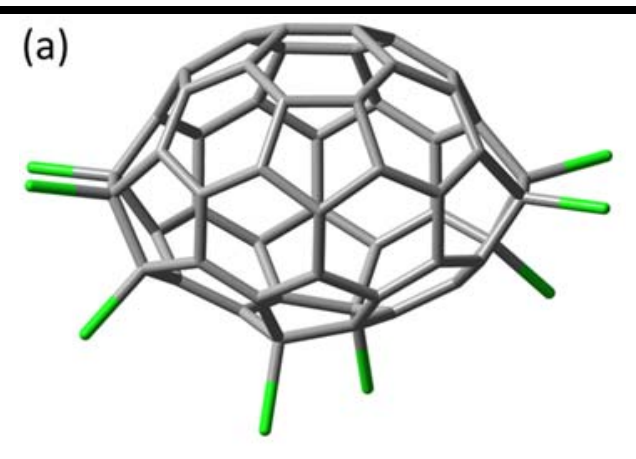

(b)

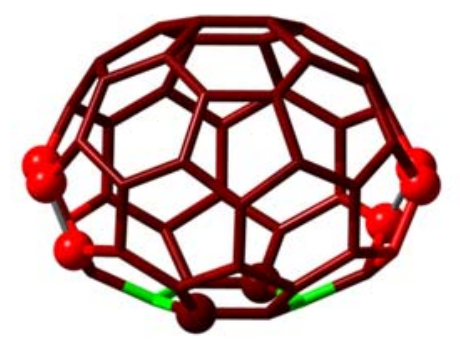

Figure 4. Crystal structure for the experimentally isolated $\mathrm{C}_{54} \mathrm{Cl}_{8}[(\mathrm{a})$, ref 59], and the corresponding $\mathrm{C}_{54}$ obtained in the present study (b). The colors in $\mathrm{C}_{54}$ represent calculated local deformation energies in the R+D model, with bright red indicating large deformations and bright green being sites of low deformation. The atoms in $\mathrm{C}_{54}$ that corresponds to the $\mathrm{C}-$ $\mathrm{Cl}$ bonding sites in $\mathrm{C}_{54} \mathrm{Cl}_{8}$ are shown as balls.

We can see that six of the eight $\mathrm{C}-\mathrm{Cl}$ bonding sites in $\mathrm{C}_{54} \mathrm{Cl}_{8}$ correspond to the carbons with the largest deformation energies. This supports the notion that sites that are most deformed are also those with the highest reactivity. Nonetheless, two remaining high-deformation sites in $\mathrm{C}_{54}$ do not correspond to the two remaining $\mathrm{C}-\mathrm{Cl}$ bonds in $\mathrm{C}_{54} \mathrm{Cl}_{8}$. A possible contributing factor to this observation could be significant repulsion between the $\mathrm{Cl}$ atoms if the additional $\mathrm{Cl}$ atoms were to occupy the remaining sites on those two five-membered rings. Interestingly, the actual sites of the last two $\mathrm{C}-\mathrm{Cl}$ bonds occur at $\mathrm{C}_{54}$ carbons next to the two with the lowest deformation energies. It is unclear if these low-deformation sites facilitate reactions at their neighboring atoms, or this observation is the result of a mere coincidence.

Case Study 2: Stability of $\mathbf{C}_{60}$. Let us now apply deformation energies to another case study. In the Introduction section, we note that $I_{h} C_{60}$ appears to have higher stability than both larger and smaller fullerenes. In our case study on $\mathrm{C}_{54}$, we highlight that sites with high 
deformation energies seem to be a reasonable indicator for high reactivities. We now apply this concept to rationalize the "special stability" of $\mathrm{C}_{60}$. For each of the fullerenes that we have examined in Figure 3, we have obtained the deformation energy for the site with the largest deformation. These maximum local-deformation-energies are shown in Figure 5, together with the corresponding average local-deformation-energies.

First of all, we note that, for $\mathrm{C}_{20}$ and $\mathrm{C}_{60}$, the maximum and average values are essentially the same. This is because, in both of these fullerenes, all atoms are essentially identical. What is more interesting is the general trend for the maximum deformation energy to decrease from $\mathrm{C}_{20}$ to $\mathrm{C}_{60}$, and then it climbs from that point onwards, eventually reaching convergence of $\sim 45 \mathrm{~kJ} \mathrm{~mol}^{-1}$. Within this set of data, an exception to such a trend is $C_{540}$. For the general trend, one can rationalize this by noting the perfectly spherical structure of $C_{60}$, such that for fullerenes that are similar in size to $\mathrm{C}_{60}$, deviations from the spherical geometry introduce sites with larger deformations (Figure 6, see also ref 10). Presumably, these highdeformation-energy sites render fullerenes on both sides of $C_{60}$ more reactive than $C_{60}$, leading to eventual accumulation of $\mathrm{C}_{60}$ as the major product in typical fullerene syntheses.

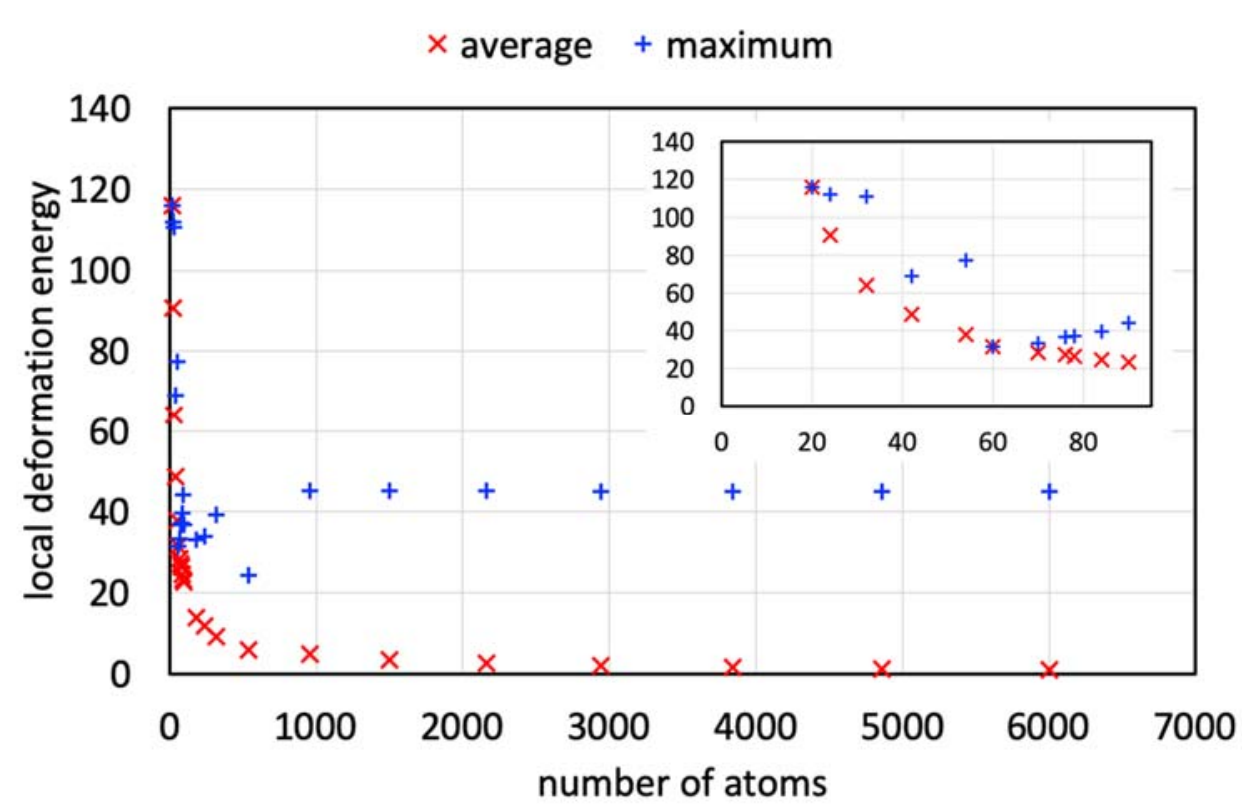

Figure 5. Average local-deformation-energy $\left(\mathrm{kJ} \mathrm{mol}^{-1}\right)$ in the $\mathrm{R}+\mathrm{D}$ model for fullerenes from $\mathrm{C}_{20}$ to $\mathrm{C}_{6000}$, and the corresponding maximum local-deformation-energy.

The $\mathrm{C}_{540}$ fullerene represents another interesting case in this set of fullerenes. Its maximum deformation energy is in fact lower than that for $\mathrm{C}_{60}$. However, it is conceivable that, under typical high-energy conditions in fullerene syntheses, entropic effect would favor 
the production of smaller fullerenes, ultimately yielding mostly $C_{60}$; downsizing from $C_{60}$ would lead to non-IPR fullerenes that are substantially less stable, both in terms of overall thermochemical stability as well as presence of high-energy local regions. Nonetheless, the relatively low value for the maximum deformation energy of $C_{540}$ hints at the possibility of producing fullerenes of such sizes if an optimal experimental condition can be achieved. If realized, such fullerenes may further the prospect of using them as, e.g., large "nano-flasks"60 for chemical and technological applications.

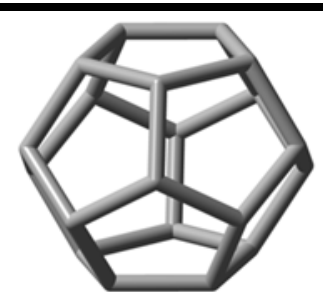

$\mathrm{C}_{20}$
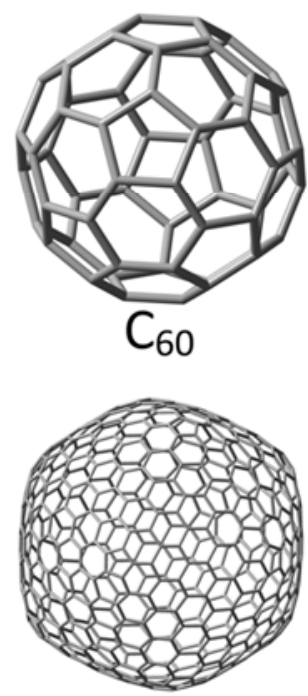

$\mathrm{C}_{540}$

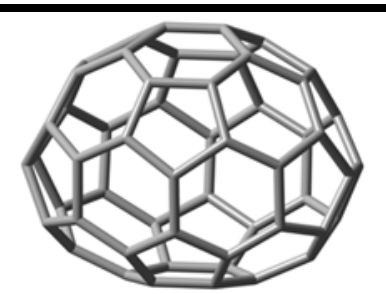

$\mathrm{C}_{54}$

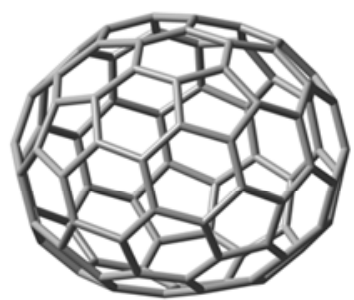

$\mathrm{C}_{90}$

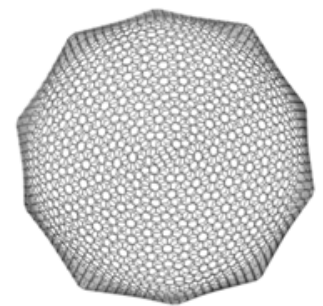

$\mathrm{C}_{6000}$

Figure 6. Structures of selected fullerenes illustrating, notably, the perfectly spherical structure of $I_{h} C_{60}$ and the pentagon vertices of giant $/ \mathrm{h}$ fullerenes $\left(C_{6000}\right)$.

At the limit of giant $/ \mathrm{h}$ fullerenes, their structures are typically characterized by sphere-like geometries with pentagon vertices and graphene-like faces (Figure 6). The sites of maximum deformation would correspond to pentagon carbons with essentially identical deformation (and hence converged maximum-deformation-energy), with differences between different giant $I_{\mathrm{h}}$ fullerenes being the size of the graphene-like faces. From this point of view, such giant fullerenes may be susceptible to downsizing, to a point where the high-energy sites can be structurally stabilized. Finally, we reiterate that our discussion is on the basis of 
deformation energy within thermochemical stability, which seems to be consistent with experimental observations discussed in our case studies. The mechanism for the formation of those eventual products in fullerene syntheses is not within the scope of the present study, but has been a subject of numerous studies (e.g., refs 61-65).

\section{CONCLUDING REMARKS}

In the present study, we have used quantum chemistry computations, in conjunction with isodesmic-type reactions, to obtain accurate heats of formation (HoFs) for several small fullerenes. Specifically, they are $2358.2 \pm 8.0 \mathrm{~kJ} \mathrm{~mol}^{-1}$ for $\mathrm{C}_{20}, 2566.2 \pm 7.6 \mathrm{~kJ} \mathrm{~mol}^{-1}$ for $\mathrm{C}_{24}$, and, for the lowest-energy isomers of $C_{32}, C_{42}$ and $C_{54}, 2461.1 \pm 15.4,2629.0 \pm 20.5$ and 2686.2 $\pm 25.3 \mathrm{~kJ} \mathrm{~mol}^{-1}$, respectively.

The good accuracy in our calculated HoFs results from several key factors. In comparison with high-level composite protocol with post-CCSD(T) terms up to $\operatorname{CCSDTQ}(5)$, we find the moderate W1X-1 method [an approximation to $\operatorname{CCSD}(T)$ at the complete-basis-set limit] capable of accurately treating the type of structural deformations in small fullerenes; we have applied W1X-1 to $C_{20}$ and $C_{24}$. Our benchmark against W1X-1 reference shows that the B97M$\mathrm{V}$ and MN15 density functionals, especially when used together as an average, provide adequate reaction energies for the kind of isodesmic-type reactions that we use for larger fullerenes; they are used for $C_{32}, C_{42}$ and $C_{54}$. To minimize uncertainties in the HoFs, for these larger fullerenes, we employ medium-sized structural motifs in the isodesmic-type reactions. Accurate HoFs for these medium-sized molecules are obtained at the W1X-1 level, the specific values are $216.6 \pm 1.4$ (fulvene, $\mathrm{C}_{6} \mathrm{H}_{6}$ ), $375.5 \pm 1.5$ (pentalene, $\mathrm{C}_{8} \mathrm{H}_{6}$ ), $670.8 \pm 2.9$ (acepentalene, $\mathrm{C}_{10} \mathrm{H}_{6}$ ), and $262.7 \pm 2.5$ (acenaphthylene, $\mathrm{C}_{12} \mathrm{H}_{8}$ ) kJ mol${ }^{-1}$.

We combine the HoFs of the small fullerenes and those previously obtained for larger fullerenes (from $\mathrm{C}_{60}$ to $\mathrm{C}_{6000}$ ) into a full picture of fullerene HoFs. The normalized (per-carbon) HoFs show an inversely proportional trend to the number of carbon atoms. The trend can be reasonably approximated by the " $R+D$ " model that we have previously developed, which takes into account the Resonance in the $\pi$-bond networks and Deformation from planarity. While $R+D$ generally approximates the HoFs well, for the smallest fullerenes $\left(C_{20}, C_{24}\right)$, there are notable deviations from the actual values. We have tentatively attributed the deviations to simplifications made in the derivation of the deformation term. 
Fullerenes that are smaller than $C_{60}$ are formally "non-IPR", i.e., they do no satisfy the isolated pentagon rule (IPR). For these small fullerenes, the energy associated with structural deformation can be expected to play an important role in their reactivity. The R+D model, where applicable, enables straightforward estimation of the local deformation energy at each atomic site of a given fullerene, and thus prediction of the local reactivity. In a brief case study, we find that, for $\mathrm{C}_{54}$, most of the high-deformation-energy carbon atoms indeed correspond to the sites of $\mathrm{C}-\mathrm{Cl}$ bond in the experimentally captured $\mathrm{C}_{54} \mathrm{Cl}_{8}$. There are, however, other factors such as repulsion between $\mathrm{Cl}$ atoms that also seems to influence stability of the product.

In another case study, we find that, for the series of fullerenes from $C_{20}$ to $C_{6000}, C_{60}$ has the lowest value for the maximum local-deformation-energy. This is consistent with the experimentally observed "special stability" for $\mathrm{C}_{60}$. Our analysis also suggests that giant fullerenes (from $\sim \mathrm{C}_{1000}$ onwards) would be prone to downsizing because they contain sites with considerable local deformations. On the other hand, the medium-size region $\left(\sim \mathrm{C}_{500}\right)$ may contain fullerenes that are fairly stable, with the possibility of being produced in prospective experimental studies.

\section{ASSOCIATED CONTENT}

\section{Supporting Information}

Additional results (PDF), geometries for the relevant species contained in a single compressed archive (ZIP).

\section{AUTHOR INFORMATION}

\section{Corresponding Author}

Bun Chan - Graduate School of Engineering, Nagasaki University, Bunkyo 1-14, Nagasaki-shi, Nagasaki 852-8521, Japan; orcid.org/0000-0002-0082-5497; E-mail: bun.chan@nagasakiu.ac.jp

\section{ACKNOWLEDGMENTS}

We gratefully acknowledge generous grants of computer time from the RIKEN Information Systems and Cybersecurity (ISC) (Project Q20266), Japan.

\section{REFERENCES}

1 Kroto, H. W.; Fischer, J. E.; Cox, D. E. The Fullerenes; Pergamon Press: Oxford, 2012.

2 Taylor, R. Lecture Notes on Fullerene Chemistry: A Handbook for Chemists; Imperial College Press: London, 1999. 
3 Schwerdtfeger, P.; Wirz, L. N.; Avery, J. The Topology of Fullerenes. WIREs Comput. Mol. Sci. 2015, 5, 96-145.

4 Osawa, E. Perspectives of Fullerene Nanotechnology; Kluwer Academic Publishers: Dordrecht, 2002.

5 Langa, F.; Nierengarten, J. F. Fullerenes: Principles and Applications, 2nd ed.; Royal Society of Chemistry: Cambridge, 2011.

6 Kubozono, Y. Physics and Chemistry of Carbon-Based Materials: Basics and Applications; Springer Nature: Singapore, 2019.

7 Karton, A.; Chan, B.; Raghavachari, K.; Radom, L. Evaluation of the Heats of Formation of Corannulene and $\mathrm{C}_{60}$ By Means of High-Level Theoretical Procedures. J. Phys. Chem. A 2013, 117, 1834-1842.

8 Chan, B.; Kawashima, Y.; Katouda, M.; Nakajima, T.; Hirao, K. From C60 to Infinity: LargeScale Quantum Chemistry Calculations of the Heats of Formation of Higher Fullerenes. J. Am. Chem. Soc. 2016, 138, 1420-1429.

9 Waite, S. L.; Chan, B.; Karton, A.; Page, A. J. Accurate Thermochemical and Kinetic Stabilities of $\mathrm{C}_{84}$ Isomers. J. Phys. Chem. A 2018, 122, 4768-4777.

10 Chan, B.; Kawashima, Y.; Dawson, W.; Katouda, M.; Nakajima, T.; Hirao, K. A Simple Model for Relative Energies of All Fullerenes Reveals the Interplay between Intrinsic Resonance and Structural Deformation Effects in Medium-Sized Fullerenes. J. Chem. Theory Comput. 2019, 15, 1255-1264.

11 Wan, W.; Karton, A. Heat of Formation for $C_{60}$ by Means of the G4(MP2) Thermochemical Protocol Through Reactions in which $\mathrm{C}_{60}$ is Broken Down into Corannulene and Sumanene. Chem. Phys. Lett. 2016, 643, 34-38.

12 Karton, A.; Waite, S. L.; Page, A. J. Performance of DFT for $\mathrm{C}_{60}$ Isomerization Energies: A Noticeable Exception to Jacob's Ladder. J. Phys. Chem. A 2019, 123, 257-266.

13 Cioslowski, J.; Rao, N.; Moncrieff, D. Standard Enthalpies of Formation of Fullerenes and Their Dependence on Structural Motifs. J. Am. Chem. Soc. 2000, 122, 8265-8270.

14 Fedorov, A. S.; Fedorov, D. A.; Kuzubov, A. A.; Avramov, P. V.; Nishimura, Y.; Irle, S.; Witek, H. A. Relative Isomer Abundance of Fullerenes and Carbon Nanotubes Correlates with Kinetic Stability. Phys. Rev. Lett. 2012, 107, 175506-1-5.

15 Sure, R.; Hansen, A.; Schwerdtfeger, P.; Grimme, S. Comprehensive Theoretical Study of All 1812 C $_{60}$ Isomers. Phys. Chem. Chem. Phys. 2017, 19, 14296-14305. 
16 American Chemical Society National Historic Chemical Landmarks. Discovery of Fullerenes.

http://www.acs.org/content/acs/en/education/whatischemistry/landmarks/fullerenes. html (accessed 8 Mar 2020).

17 Frisch, M. J.; Trucks, G. W.; Schlegel, H. B.; Scuseria, G. E.; Robb, M. A.; Cheeseman, J. R.; Scalmani, G.; Barone, V.; Petersson, G. A.; Nakatsuji, H., et al. Gaussian 16, Revision A.03; Gaussian, Inc.: Wallingford CT, 2016.

18 Werner, H.-J.; Knowles, P. J.; Knizia, G.; Manby, F. R.; Schütz, M. Molpro: A GeneralPurpose Quantum Chemistry Program Package. WIREs Comput. Mol. Sci. 2012, 2, 242253.

19 Kállay, M.; Nagy, P. R.; Mester, D.; Rolik, Z.; Samu, G.; Csontos, J.; Csóka, J.; Szabó, P. B.; Gyevi-Nagy, L.; Hégely, B., et al. The MRCC Program System: Accurate Quantum Chemistry from Water to Proteins. J. Chem. Phys. 2020, 152, 074107-1-18.

20 Shao, Y.; Gan, Z.; Epifanovsky, E.; Gilbert, A. T. B.; Wormit, M.; Kussmann, J.; Lange, A. W.; Behn, A.; Deng, J.; Feng, X., et al. Advances in Molecular Quantum Chemistry Contained in the Q-Chem 4 Program Package. Mol. Phys. 2015, 113, 184-215.

21 Schwerdtfeger, P.; Wirz, L. N.; Avery, J. Fullerene - a Software Package for Constructing and Analyzing Structures of Regular Fullerenes. J. Comput. Chem. 2013, 34, 1508-1526.

22 Aihara, J. A New Definition of Dewar-Type Resonance Energies. J. Am. Chem. Soc. 1976, $98,2750-2758$.

23 Gutman, I.; Milorad, M.; Nenad, T. Graph Theory and Molecular Orbitals. 19. Nonparametric Resonance Energies of Arbitrary Conjugated Systems. J. Am. Chem. Soc. $1977,99,1692-1704$.

24 Guan, J.; Jin, Z.; Zhu, Z.; Chuang, C.; Jin, B.-Y.; Tománek, D. Local Curvature and Stability of Two-Dimensional Systems. Phys. Rev. B 2014, 90, 245403-1-6.

25 Stephens, P. J.; Devlin, F. J.; Chabalowski, C. F.; Frisch, M. J. Ab Initio Calculation of Vibrational Absorption and Circular Dichroism Spectra Using Density Functional Force Fields. J. Phys. Chem. 1994, 98, 11623-11627.

26 Dunning, T. H. Jr. Gaussian Basis Sets for Use in Correlated Molecular Calculations. I. The Atoms Boron Through Neon and Hydrogen. J. Chem. Phys. 1989, 90, 1007-1023.

27 Chan, B.; Radom, L. W1X-1 and W1X-2: W1-Quality Accuracy with an Order of Magnitude Reduction in Computational Cost. J. Chem. Theory Comput. 2012, 8, 4259-4269. 
28 Chan, B.; Radom, L. W3X: A Cost-Effective Post-CCSD(T) Composite Procedure. J. Chem. Theory Comput. 2013, 9, 4769-4778.

29 Chan, B.; Radom, L. W2X and W3X-L: Cost-Effective Approximations to W2 and W4 with kJ mol-1 Accuracy. J. Chem. Theory Comput. 2015, 11, 2109-2119.

30 Chan, B. How to Computationally Calculate Thermochemical Properties Objectively, Accurately, and as Economically as Possible. Pure Appl. Chem. 2017, 89, 699-713.

31 Chan, B. Unification of the W1X and G4(MP2)-6X Composite Protocols. J. Chem. Theory Comput. 2017, 13, 2642-2649.

32 Chan, B.; Radom, L. Frequency Scale Factors for Some Double-Hybrid Density Functional Theory Procedures: Accurate Thermochemical Components for High-Level Composite Protocols. J. Chem. Theory Comput. 2016, 12, 3774-3780.

33 Chan, B.; Use of Low-Cost Quantum Chemistry Procedures for Geometry Optimization and Vibrational Frequency Calculations: Determination of Frequency Scale Factors and Application to Reactions of Large Systems. J. Chem. Theory Comput. 2017, 13, 6052-6060.

34 Mardirossian, N.; Head-Gordon, M. Mapping the Genome of Meta-Generalized Gradient Approximation Density Functionals: The Search for B97M-V. J. Chem. Phys. 2015, 142, 074111-1-32.

35 Yu, H. S.; He, X.; Li, S. L.; Truhlar, D. G. MN15: A Kohn-Sham Global-Hybrid ExchangeCorrelation Density Functional with Broad Accuracy for Multi-Reference and SingleReference Systems and Noncovalent Interactions Chem. Sci. 2016, 7, 5032-5051.

36 Papajak, E.; Leverentz, H. R.; Zheng, J.; Truhlar, D. G. Efficient Diffuse Basis Sets: cc-pVxZ+ and maug-cc-pVxZ. J. Chem. Theory Comput. 2009, 5, 1197-1202.

37 Chan, B.; Deng, J.; Radom, L. G4(MP2)-6X: a Cost-Effective Improvement to G4(MP2). J. Chem. Theory Comput. 2010, 7, 112-120.

38 Chan, B.; Karton, A.; Raghavachari, K. G4(MP2)-XK: A Variant of the G4(MP2)-6X Composite Method with Expanded Applicability for Main-Group Elements up to Radon. J. Chem. Theory Comput. 2019, 15, 4478-4484.

39 Montgomery Jr., J. A.; Frisch, M. J.; Ochterski, J. W.; Petersson, G. A. A Complete Basis Set Model Chemistry. VII. Use of the Minimum Population Localization Method. J. Chem. Phys. 2000, 112, 6532-6542. 
40 Chan, B.; Radom, L. Obtaining Good Performance with Triple- $\zeta$-Type Basis Sets in DoubleHybrid Density Functional Theory Procedures. J. Chem. Theory Comput. 2011, 7, 28522863.

41 Pople, J. A.; Head-Gordon, M.; Raghavachari, K. Quadratic Configuration Interaction - A General Technique for Determining Electron Correlation Energies. J. Chem. Phys. 1987, $87,5968-5975$.

42 Bakowies, D. Estimating Systematic Error and Uncertainty in Ab Initio Thermochemistry: II. ATOMIC(hc) Enthalpies of Formation for a Large Set of Hydrocarbons. J. Chem. Theory Comput. 2020, 16, 399-426.

43 Lee, J.; Head-Gordon, M. Distinguishing Artificial and Essential Symmetry Breaking in a Single Determinant: Approach and Application to the $C_{60}, C_{36}$, and $C_{20}$ Fullerenes. Phys. Chem. Chem. Phys. 2019, 21, 4763-4778.

44 Manna, D.; Martin, J. M. L. What Are the Ground State Structures of $C_{20}$ and $C_{24}$ ? An Explicitly Correlated Ab Initio Approach. J. Phys. Chem. A 2016, 120, 153-160.

45 Karton, A.; Rabinovich, E.; Martin, J. M. L.; Ruscic, B. W4 Theory for Computational Thermochemistry: In Pursuit of Confident Sub-kJ/mol Predictions. J. Chem. Phys. 2006, $125,144108-1-17$.

46 Halkier, A.; Helgaker, T.; Jørgensen, P.; Klopper, W.; Koch, H.; Olsen, J.; Wilson. A. K. BasisSet Convergence in Correlated Calculations on $\mathrm{Ne}, \mathrm{N}_{2}$, and $\mathrm{H}_{2} \mathrm{O}$. Chem. Phys. Lett. 1998, 286, 243-252.

47 Douglas, M.; Kroll, N. M. Quantum Electrodynamical Corrections to Fine-Structure of Helium. Ann. Phys. 1974, 82, 89-155.

48 Hess, B. A. Applicability of the No-Pair Equation with Free-Particle Projection Operators to Atomic and Molecular-Structure Calculations. Phys. Rev. A 1985, 32, 756-763.

49 Ruscic, B.; Pinzon, R. E.; von Laszewski, G.; Kodeboyina, D.; Burcat, A.; Leahy, D.; Montoya, D.; Wagner, A. F. Active Thermochemical Tables: Thermochemistry for the 21st Century. J. Phys. Conf. Ser. 2005, 16, 561-570.

$50 \mathrm{Ku}, \mathrm{H} . \mathrm{H}$. Notes on the Use of Propagation of Error Formulas. J. Res. Natl. Bur. Stand. 1966, 70C, 263-273.

51 Wheeler, S. E.; Houk, K. N.; Schleyer, P. v. R.; Allen, W. D. A Hierarchy of Homodesmotic Reactions for Thermochemistry. J. Am. Chem. Soc. 2009, 131, 2547-2560. 
52 NIST Chemistry WebBook; Linstrom, P. J.; Mallard, W. G., Eds; NIST Standard Reference Database Number 69; National Institute of Standards and Technology: Gaithersburg MD, http://webbook.nist.gov, (retrieved 30 Mar 2020).

53 Slayden, S. W.; Liebman, J. F. The Energetics of Aromatic Hydrocarbons: An Experimental Thermochemical Perspective. Chem. Rev. 2001, 101, 1541-1566.

54 Chan, B. Formulation of Small Test Sets Using Large Test Sets for Efficient Assessment of Quantum Chemistry Methods. J. Chem. Theory Comput. 2018, 14, 4254-4262.

55 Chan, B.; Gill, P. M. W.; Kimura, K. Assessment of DFT Methods for Transition Metals with the TMC151 Compilation of Data Sets and Comparison with Accuracies for Main-Group Chemistry. J. Chem. Theory Comput. 2019, 15, 3610-3622.

56 Chan, B. The CUAGAU Set of Coupled-Cluster Reference Data for Small Copper, Silver, and Gold Compounds and Assessment of DFT Methods. J. Phys. Chem. A 2019, 123, 57815788.

57 Noël, Y.; De La Pierre, M.; Zicovich-Wilson, C. M.; Orlando, R.; Dovesi, R. Structural, Electronic and Energetic Properties of Giant Icosahedral Fullerenes Up to $\mathrm{C}_{6000}$ : Insights from an Ab Initio Hybrid DFT Study. Phys. Chem. Chem. Phys. 2014, 16, 13390-13401.

58 Kroto, H. W. The stability of the Fullerenes $C_{n}$, with $n=24,28,32,36,50,60$ and 70. Nature 1987, 329, 529-531.

59 Tan, Y.-Z.; Li, J.; Zhu, F.; Han, X.; Jiang, W.-S.; Huang, R.-B.; Zheng, Z.; Qian, Z.-Z.; Chen, R.T.; Liao, Z.-J., et al. Chlorofullerenes Featuring Triple Sequentially Fused Pentagons. Nat. Chem. 2010, 2, 269-273.

60 Morinaka, Y.; Zhang, R.; Sato, S.; Nikawa, H.; Kato, T.; Furukawa, K.; Yamada, M.; Maeda, Y.; Murata, M.; Wakamiya, A., et al. Fullerene $C_{70}$ as a "Nano-Flask" that Reveals the Chemical Reactivity of Atomic Nitrogen. Angew. Chem. Int. Ed. 2017, 56, 6488-6491.

61 Irle, S.; Zheng, G.; Wang, Z.; Morokuma, K. The $C_{60}$ Formation Puzzle "Solved": QM/MD Simulations Reveal the Shrinking Hot Giant Road of the Dynamic Fullerene Self-Assembly Mechanism. J. Phys. Chem. B 2006, 110, 14531-14545.

62 Saha, B.; Irle, S.; Morokuma, K. Hot Giant Fullerenes can Eject and Capture $C_{2}$ Molecules: QM/MD Simulations with Constant Density. J. Phys. Chem. C 2011, 115, 22707-22716.

63 Zhang, J.; Bowles, F. L.; Bearden, D. W.; Ray, W. K.; Fuhrer, T.; Ye, Y.; Dixon, C.; Harich, K.; Helm, R. F.; Olmstead, M. M., et al. A Missing Link in the Transformation from Asymmetric 
to Symmetric Metallofullerene Cages Implies a Top-Down Fullerene Formation Mechanism. Nat. Chem. 2013, 5, 880-885.

64 Dunk, P. W. Bottom-Up Formation of Endohedral Mono-Metallofullerenes is Directed by Charge Transfer. Nat. Commun. 2014, 5, 5844-1-8.

65 Qian, H.-J.; Wang, Y.; Morokuma, K. Quantum Mechanical Simulation Reveals the Role of Cold Helium Atoms and the Coexistence of Bottom-Up and Top-Down Formation Mechanisms of Buckminsterfullerene from Carbon Vapor. Carbon 2017, 114, 635-641. 
TOC GRAPHICS

fullerene thermochemistry

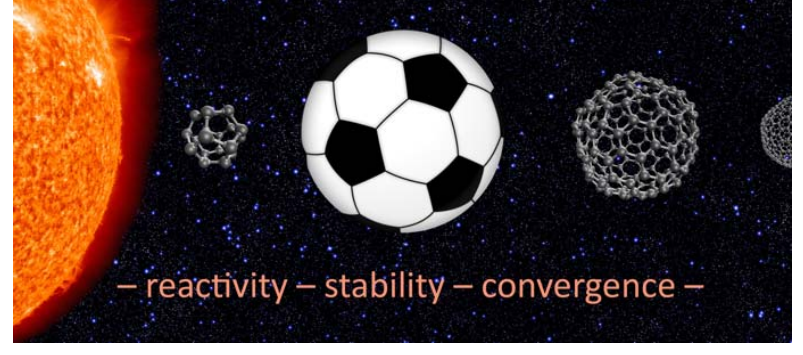

